\title{
What the clinician needs to know about magic mushrooms
}

\section{Nicholas Seivewright \& Olawale Lagundoye}

\section{What are magic mushrooms?}

This term refers to mushrooms that grow naturally and have hallucinogenic (sometimes called psychedelic) properties. Consumption of different species has occurred in various cultures over the centuries, with use in ritual ceremonies in Mexico being particularly well known. In the UK at present the species most commonly used is Psilocybe semilanceata, also known as the 'liberty cap' mushroom. This grows in many areas, particularly in dark places and after heavy rainfall, with fruition occurring from September to November. It is creamyyellow or brown in colour, very small (5-15 mm across) with a thin fragile stalk. In the USA a closely related type is used.

The active chemical psilocybin is categorised in class A in the UK's Misuse of Drugs Act 1971, so in theory there can be severe penalties for offences. However, possession of the mushrooms in their natural state is not illegal; charges can be brought only if they have been prepared in some way for consumption (see below).

\section{Usage}

Psilocybe semilanceata mushrooms (to which the rest of this article will refer) can be eaten raw, or cooked or brewed into a liquid. They may also be dried, sometimes for keeping for later use, and the dried form can be put into cigarettes, a pipe, or home-made capsules. There have apparently been rare instances of injecting the liquid form.

Because the mushrooms are small, about 10-100 are typically used at a time. Nearly all usage is personal or within small groups, and there is no significant illicit market. They are commonly used experimentally by young people, with the recent British Crime Survey (Home Office, 1999) showing that just under 1 in 10 16- to 29-year-olds had tried magic mushrooms. Some experimenters become more regular users, with older cases often being individuals with somewhat alternative lifestyles. The most common other substances of misuse in mushroom users appear to be alcohol and cannabis. In general, mushrooms are consumed on their own.

In no sense can magic mushrooms be said to be truly addictive, with one particular measure finding them to be the least dependence-producing of all illicit substances (Gable, 1993). However, a degree of tolerance does occur, so that if they are consumed on two days running more will be required the second time to achieve the desired effects. This tolerance disappears within days, but effectively acts as a natural constraint against very frequent use.

\section{Desired effects}

It is well known that the effects of magic mushrooms broadly resemble those of lysergic acid diethylamide (LSD) (Leikin et al, 1989). The desired effects, which to some extent relate to the user's expectations, include a pleasant state of detachment and euphoria and then the hallucinatory experiences - more

Nicholas Seivewright is Consultant Psychiatrist in Substance Misuse at Community Health Sheffield NHS Trust. His main interests are in personality disorder and the use of pharmacological treatments in drug misuse. Olawale Lagundoye is Specialist Registrar in Substance Misuse in Sheffield. He is particularly interested in transcultural aspects of psychiatry and in dual diagnosis. 
correctly, often illusions or pseudo-hallucinations, involving images, colours and sounds. In a report of cases seen in the early days of magic mushroom use in this country (Peden et al, 1981), distortions of perception of faces received special comment. The phenomenon of synaesthesia, in which sensations cross between modalities so that for instance colours may appear to have a smell, has mainly been reported with LSD, but may possibly occur with mushrooms. The overall experience is referred to as a 'trip', which starts within 20 minutes or so of ingestion and may last several hours, partly dependent on the amount taken and the route.

The effects of hallucinogenic drugs generally appear to be partly related to actions on the serotonergic transmission system, but the mechanisms relating to mushrooms in particular are not known.

\section{Adverse effects}

The possible adverse effects of magic mushrooms are summarised in Box 1.

\section{Physical}

If many mushrooms are taken, the user can experience nausea, vomiting, stomach pains and dizziness. There is general physiological arousal, with tachycardia more frequent than elevations in blood pressure (Peden et al, 1981). Pupils become enlarged but react to light. The main physical danger, however, occurs if other species of similar-looking poisonous mushrooms are taken by mistake, such as the Amanita species in the UK. Some forms can be fatal, and identification by a mycologist may be necessary if the mushrooms are available. Also, accidents of various kinds may occur in states of intoxication.

\section{Psychological}

Any hallucinogenic drug can produce a so-called 'bad trip' instead of the desired effects. In this there is anxiety and general mood disturbance, with the hallucinations and alterations in consciousness seeming alarming. This may occur in inexperienced users, or when dysphoria was present before taking the drug. Acute confusional states may develop, while the hallucinatory experiences can develop into a state that it is correct to term a psychotic disorder. Such states would be expected to subside completely within about 24 hours at the maximum, but the clinical picture may be complicated if other drugs such as alcohol or cannabis have been used.
Box 1 Adverse effects of magic mushrooms

Nausea and vomiting

Tachycardia

Poisoning (if 'wrong' species taken)

Accidents

Anxiety, panic

Acute confusion

Psychotic reactions

The prototype of the hallucinogenic drugs in terms of effects and adverse effects is LSD, the psychiatric complications of which have been reviewed by Abraham \& Aldridge (1993). It can be supposed that magic mushrooms produce modified forms of some of these typical effects, but it must be recognised that very little literature refers to mushrooms specifically.

\section{Testing}

Testing for psilocybin is not included in the routine urine (or hair) screening for drugs done by UK hospital laboratories. Special assays can be set up, but the short half-life of the chemical means that detection would be missed in some cases. Urine screening for the range of substances is, however, always clinically indicated where drug use is suspected, and clearly mushroom users may have used other substances. Also, it appears that sometimes non-hallucinogenic mushrooms are 'spiked' with drugs such as LSD.

\section{Classification of disorders}

The ICD-10 (World Health Organization, 1992) specifies mental and behavioural disorders due to psychoactive substance use, which may be applied to hallucinogens as one of nine categories of drugs (there is also a coding for disorders due to multiple drug use). Box 2 shows the diagnoses that could possibly be made in relation to magic mushrooms.

\section{Management}

As seen currently in the UK the use of magic mushrooms is either experimental in nature or represents a kind of lifestyle feature, and users would hardly 
Box 2 Possible diagnoses in use of magic mushrooms

F16.0 - Acute intoxication

F16.1 - Harmful use

F16.5 - Psychotic disorder

ever view themselves as having a problem with the drug, with the possible exception of some of the acute effects. It is, therefore, virtually unheard of for users to present to drug services asking for help with cutting down or stopping, although such advice could be given if necessary. In practice, the relevant management is of complications such as toxicity or confusional or brief psychotic states, and is largely supportive with symptomatic treatment as necessary. There are no features of mushroom use that would lead to selection of specific treatments within such management.

\section{Conclusions}

The main clinical messages regarding magic mushrooms are indicated in Box 3 .

Whenever psychiatrists encounter cases of drug misuse, consideration must be given to possible additional diagnoses. The strongest overall association is with personality disorder (Seivewright \& Daly, 1997), with studies indicating that about twothirds of drug misusers have this diagnosis, usually the antisocial category. However, most studies are of opiate misusers in treatment settings, and it is reasonable to assume a lower rate in users of the more 'recreational' drugs, although such use may

\section{Box 3 Main learning points}

Magic mushrooms are not addictive, but produce physiological effects and sometimes significant psychiatric complications

Although 'recreational', the use of magic mushrooms may be one indicator of conduct or personality disorder

Urine testing cannot usually detect the psilocybin constituent of mushrooms, but should be done to screen for other drugs

Management of complications is symptomatic, with no specific approaches indicated still be an indicator of personal problems of various kinds.

Clinicians will rarely be called upon to advise users regarding their magic mushroom use, but knowledge is necessary of the possible direct psychiatric complications and the occasional need for management.

\section{References}

Abraham, H. D. \& Aldridge, A. M. (1993) Adverse consequences of lysergic acid diethylamide. Addiction, 88, 1327-1334.

Gable, R. S. (1993) Toward a comparative overview of dependence potential and acute toxicity of psychoactive substances used non-medically. Journal of Drug and Alcohol Abuse, 19, 263-281.

Home Office (1999) Drug Misuse Declared in 1998: Results from the British Crime Survey. London: Home Office.

Leikin, J. B., Krantz, A. J., Zell-Kanter, M., et al (1989) Clinical features and management of intoxication due to hallucinogenic drugs. Medical Toxicology and Adverse Drug Experience, 4, 324-350.

Peden, N. R., Bisset, A. F., Macaulay, K. E. C., et al (1981) Clinical toxicology of "magic mushrooms" ingestion. Postgraduate Medical Journal, 57, 543-545.

Seivewright, N. \& Daly, C. (1997) Personality disorder and drug use: a review. Drug and Alcohol Review, 16, 235-250.

World Health Organization (1992) The ICD-10 Classification of Mental and Behavioural Disorders. Clinical Descriptions and Diagnostic Guidelines. Geneva: WHO.

\section{Multiple choice questions}

1. Psilocybin:

a is the active chemical in a wild mushroom commonly used in the UK

b has predominantly sedative effects

c is classified under the Misuse of Drugs Act 1971

$\mathrm{d}$ is detectable in routine urine drug screening

e produces psychological effects that mostly last for less than one hour.

2. The following are recognised features of magic mushroom use:

a use of a dried preparation by smoking

b bradycardia

c acute confusional states

$\mathrm{d}$ experimental use by young people

e a short-lived tolerance effect.

3. The psychiatric effects of magic mushrooms:

a are more severe than those of LSD

b may include auditory hallucinations

c frequently recover without medication

d include depression as a characteristic longterm feature

e may partly constitute what is colloquially known as a 'bad trip'. 
4. Magic mushroom users in the UK:

a use the drug mainly in autumn

b typically take mushrooms together with amphetamine

c represent a major public health problem

d may be found to have conduct disorder if they use mushrooms in early teenage years

e are seen in large numbers by drug treatment services.

5. Hallucinogenic drugs:

a are regularly used by most active heroin users

b include amphetamine

c may lead to accidents due to intoxication d can produce physical dependence

e are the class of drugs most associated with 'flashback' experiences.

MCQ answers

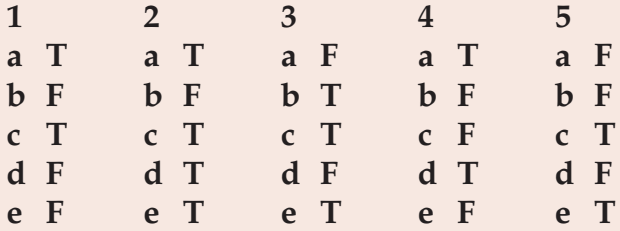

\section{New from Gaskell}

\section{Liaison Psychiatry: Planning Services for Specialist Settings}

\section{R. Peveler, E. Feldman and T. Friedman}

Liaison psychiatry services have the potential to improve patient care and streamline medical services but are underdeveloped in many European health systems. This book follows on from the 1994 publication 'Liaison Psychiatry: defining needs and planning services' and is planned as a practical guide to the development of services for a range of specialist settings. These include services in obstetrics, A\&E, intensive care, pain clinics and trauma services.

In addition there is guidance on the general principles of developing a case of need for services, and working with purchasers or commissioners of health care. This book will be of particular value to clinicians seeking to develop services in these areas, and those seeking evidence on the value of such services.

\section{Features}

- Practical guide to estimating needs and planning services for aspects of general hospital psychiatry.

- Comprehensive assessment of evidence from the existing literature.

- How best to make a case of need to purchasers/commissioners of health care.

\section{September/October 2000, paperback, £30.00, ISBN 1901242471}

Book Sales, The Royal College of Psychiatrists, 17 Belgrave Square, London SW1X 8PG. Tel: +44 (0)20 72352351 ext. 146; Fax: +44 (0)20 72451231

The latest information on College publications is on the Internet at: www.rcpsych.ac.uk 